\title{
Cerebral Venous Sinus Thrombosis Associated with COVID-19: A Case Series and Literature Review
}

\section{Vahid Reza Ostovan}

Shiraz University of Medical Sciences

\section{Razieh Foroughi}

Shiraz University of Medical Sciences

\section{Mahtab Rostami}

Shiraz University of Medical Sciences

\section{Mostafa Almasi-Dooghaee}

Iran University of Medical Sciences

\section{Manouchehr Esmaili}

Urmia University of Medical Sciences

\section{Ali Akbar Bidaki}

Shiraz University of Medical Sciences

\section{Zahra Behzadi}

Shiraz University of Medical Sciences

Farzane Farzadfard

Shiraz University of Medical Sciences

Hoda Marbooti

Shiraz University of Medical Sciences

Abbas Rahimi-Jaberi

Shiraz University of Medical Sciences

Maryam Poursadeghfard

Shiraz University of Medical Sciences

\section{Nima Fadakar}

Shiraz University of Medical Sciences

\section{Mahnaz Bayat}

Shiraz University of Medical Sciences

\section{Maryam Owjfard}

Shiraz University of Medical Sciences

\section{Mohammad Saied Salehi}

Shiraz University of Medical Sciences

\section{Seyedeh Shaghayegh Zafarmand}

Shiraz University of Medical Sciences 
Urmia University of Medical Sciences

\section{Anahid Safari}

Shiraz University of Medical Sciences

\section{Shima Shahjouei}

Geisinger Health System

\section{Ashkan Mowla}

University of Southern California

\section{Reza Azarpazhooh}

University of Southern California

\section{Ramin Zand}

Geisinger Health System

Etrat Hooshmandi ( $\square$ ehoshmandi@gmail.com )

Shiraz University of Medical Sciences

Afshin Borhani-Haghighi ( $\square$ neuro.ab@gmail.com )

Shiraz University of Medical Sciences

\section{Research Article}

Keywords: SARS-CoV-2, COVID-19, Coronavirus, Cerebral Venous Sinus Thrombosis

Posted Date: November 30th, 2020

DOI: https://doi.org/10.21203/rs.3.rs-115097/v1

License: (c) (1) This work is licensed under a Creative Commons Attribution 4.0 International License.

Read Full License 


\section{Cerebral Venous Sinus Thrombosis Associated with COVID-19: A Case Series and Literature Review}

Vahid Reza Ostovan ${ }^{1 \#}$, Razieh Foroughi ${ }^{1 \#}$, Mahtab Rostami ${ }^{1 \#}$, Mostafa Almasi-Dooghaee ${ }^{2}$, Manouchehr Esmaili ${ }^{3}$, Ali Akbar Bidaki ${ }^{1}$, Zahra Behzadi ${ }^{1}$, Farzane Farzadfard ${ }^{1}$, Hoda Marbooti ${ }^{1}$, Abbas Rahimi-Jaberi ${ }^{1}$, Maryam Poursadeghfard ${ }^{1}$, Nima Fadakar ${ }^{1}$, Mahnaz Bayat ${ }^{1}$, Maryam Owjfard ${ }^{1}$, Mohammad Saied Salehi ${ }^{1}$, Seyedeh Shaghayegh Zafarmand ${ }^{1}$, Farzad Mardi ${ }^{3}$, Anahid Safari ${ }^{4}$, Shima Shahjouei ${ }^{5}$, Ashkan Mowla ${ }^{6}$, M Reza Azarpazhooh ${ }^{7}$, Ramin Zand ${ }^{8}$, Etrat Hooshmandi ${ }^{*}{ }^{*}$, Afshin Borhani-Haghighi ${ }^{*}{ }^{*}$

1. Clinical Neurology Research Center, Shiraz University of Medical Sciences, Shiraz, Iran.

2. Neurology Department, Firoozgar Hospital, Iran University of Medical Sciences, Tehran, Iran.

3. Neurology Department, Naghadeh Hospital, Urmia University of Medical Sciences, Urmia, Iran.

4. Stem Cells Technology Research Center, Shiraz University of Medical Sciences, Shiraz, Iran.

5. Neurology Department, Neuroscience Institute, Geisinger Health System, Danville, PA, USA.

6. Division of Stroke and Endovascular Neurosurgery, Department of Neurological Surgery, Keck School of Medicine, University of Southern California, California, USA.

7. Department of Clinical Neurological Sciences and Stroke Prevention \& Atherosclerosis Research Center, Western University, London, Canada.

8. Neuroscience Institute, Geisinger Health System, Danville, PA, USA.

\#Vahid Reza Ostovan, Razieh Foroughi, and Mahtab Rostami have contributed equally to this work and should be considered as co-first authors.

Running Title: Cerebral Venous Sinus Thrombosis and SARS-CoV-2 infection

\section{*Corresponding authors:}

Etrat Hooshmandi and Afshin Borhani-Haghighi

Clinical Neurology Research Center, Shiraz University of Medical Sciences,

P.O.Box: 7193635899, Shiraz, Iran.

Tel/Fax: +98-713-6281572

E-mail addresses: ehoshmandi@gmail.com (E. Hooshmandi), neuro.ab@gmail.com (A. BorhaniHaghighi) 


\section{Abstract}

Background: Since the COVID-19 pandemic, several cases of cerebral venous sinus thrombosis (CVST) have been reported in SARS-CoV-2 infected individuals. This study provides a series of patients with CVST and SARS-CoV-2 infection.

Methods: Consecutive patients with documented SARS-CoV-2 infection, as well as clinical and radiological characteristics of CVST, were reported from three teaching hospitals in the South West, North West, and the center of Iran from June to July 2020. We also searched the abstract archives until the end of August 2020 and gathered 28 reported cases. The diagnostic criteria for SARS-CoV-2 infection were determined according to SARS-CoV-2 detection in oropharyngeal or nasopharyngeal samples in clinically suspected patients. Demographics, main COVID-19 symptoms, confirmatory tests for SARS-CoV-2 infection diagnosis, the interval between the diagnosis of SARS-CoV-2 infection and CVST, clinical and radiological features of CVST, therapeutic strategies, CVST outcomes, rate of hemorrhagic transformation, and mortality rate were investigated.

Results: Six patients (aged 31 to 62 years old) with confirmed CVST and SARS-CoV-2 infection were admitted to our centers. Four patients had no respiratory symptoms of SARS-CoV-2 infection. Five out of six patients developed the clinical manifestations of CVST and SARS-CoV2 infection simultaneously. Three patients had known predisposing factors for CVST. Despite receiving CVST and SARS-CoV-2 infection treatments, four out of six patients passed away.

Conclusions: The role of SARS-CoV-2 as a "cause" versus an "additive contributor" remains to be elucidated. Practitioners should be aware of the possibility of CVST in SARS-CoV-2 infection.

Keywords: SARS-CoV-2; COVID-19; Coronavirus; Cerebral Venous Sinus Thrombosis 


\section{Introduction}

Cerebral venous sinus thrombosis (CVST) is far less common than arterial stroke [1]; however, it occupies a prominent place in the field of cerebrovascular medicine due to the involvement of young adults and women of reproductive age [2, 3]. Furthermore, acute-phase survival and favorable long-term prognosis depend strongly on receiving prompt and adequate treatment. This makes early diagnosis of CVST tremendously important [4].

Since the evolution of the Coronavirus Disease 2019 (COVID-19) pandemic, non-respiratory manifestations of the disease like neurological manifestations [5] and thrombotic events have been progressively appreciated. Although there are some case reports and one case series [6-18] showing the association of the CVST and SARS-CoV-2 infection, the data about diagnosis and prognosis in this association is still limited. We investigated the demographic, clinical, radiological, and prognostic characteristics of this association to improve the diagnosis and treatment of patients with CVST and COVID-19.

\section{Methods}

Consecutive patients with documented SARS-CoV-2 infection, as well as clinical and radiological characteristics of CVST, were recruited from three teaching hospitals in the Southwest, Northwest, and the center of Iran from June to July 2020. Only patients with either simultaneous CVST and COVID-19 presentation or CVST after two weeks of the typical presentations of COVID-19 were included. Patients who were admitted to the hospital with a diagnosis of CVST and developed COVID-19 as a nosocomial infection were excluded.

The diagnostic criteria for COVID-19 were determined according to SARS-CoV-2 detection in oropharyngeal or nasopharyngeal samples in clinically suspected patients [19].

CVST diagnosis was performed according to the American Heart Association/American Stroke Association statement [20]. Criteria for the diagnosis of CVST were the presence of relevant neurological syndromes (headache, intracranial hypertension, focal neurologic deficits, and/or encephalopathic states), and radiological confirmation (computed tomography (CT), CT venography, brain magnetic resonance imaging (MRI), and/or MR venography). Patients, whose manifestations could be better explained by any other neurological diseases were excluded. 
We collected a wide range of variables including, sociodemographics and vascular risk factors. We also assessed information, COVID-19 symptoms, confirmatory tests for SARS-CoV-2 infection diagnosis, the interval between the clinical suspicion of SARS-CoV-2 infection and CVST onset. Clinical and radiological characteristics of CVST, including areas of involvement, therapeutic strategies, and outcomes were collected for all cases.

The possible predisposing factors for CVST were categorized into hormone-related causes (pregnancy, post-partum and oral contraceptive use), dehydration (including religious fasting), obesity, mechanical and traumatic causes, infections rather than SARS-CoV-2, hereditary thrombophilia (particularly proteins $\mathrm{C}$ and S deficiency, factor V Leiden, antithrombin III deficiency, hyperhomocysteinemia), malignancies, hematologic disorders (particularly sickle cell disease and paroxysmal nocturnal hemoglobinuria), inflammatory disorders (collagen vascular diseases, Behcet disease, vasculitis, inflammatory bowel disease), drug-induced, multifactorial, and idiopathic.

We also searched the abstract archives including PubMed, Web of Science, Scopus, and Google Scholar published until the end of August 2020. Searches were conducted using the following MeSH terms: "SARS-CoV-2", "COVID-19", "Cerebral Venous Sinus Thrombosis", and “Cerebral Venous Thrombosis" and relevant full texts were found.

This study was approved by the ethics committee and institutional review board of Shiraz University of Medical Sciences (IR.sums.rec.1399-235).

\section{Results}

Eight patients were initially recruited. In two patients, a diagnosis of SARS-CoV-2 infection was suspected due to the presence of typical findings in chest high-resolution computerized tomography or the presence of SARS-CoV-2-specific IgM antibody. For the sake of the highest specificity, these patients were excluded. Six patients with CVST and SARS-CoV-2 infection were

presented. Demographics, main COVID-19 symptoms, confirmatory tests for COVID-19 diagnosis, the interval between the clinical suspicion to COVID-19 and the diagnosis of CVST, clinical and radiological characteristics of CVST, therapeutic strategies, and outcomes of patients 
with CVST associated with SARS-CoV-2 infection were investigated and presented in Table 1. Four out of six patients had no respiratory symptoms of SARS-CoV-2 infection. Five out of six patients had the clinical manifestations of CVST and SARS-CoV-2 infection, simultaneously. Three patients had known predisposing factors for CVST including pregnancy, protein C deficiency, and Hepatitis C. One patient had a previous history of CVST following an oral contraceptive usage. One patient was not a known case of systemic lupus erythematosus (SLE) or antiphospholipid antibody syndrome (APLS) but showed positive lupus anticoagulant during SARS-CoV-2 infection. Despite receiving CVST and COVID-19 treatments, four out of six patients died (between the day of admission until 21 days after admission). In two patients, death was primarily due to neurological deterioration and in two patients, death was primarily due to COVID-19. Endovascular intervention was performed for one of our patients; this proved to be futile despite initial recanalization. Case no.1, as an illustrative case, is presented in detail. The clinical vignettes of the other patients (case no.2-6) were provided in the supplementary file.

A cumulative data was defined as an amalgam of the current series and the previously reported cases [6-18]. A comparison between this cumulative data and some large-volume CVST studies performed before the COVID-19 pandemic [1, 3, 4, 21, 22], has been made (Table 3).

\section{Illustrative patient}

A 62-year-old Iranian woman was admitted to the Emergency Department of Namazi Hospital, Shiraz, in the southern part of Iran in June 2020. She reported a 5-day history of a gradual and progressive throbbing right-sided occipital headache associated with nausea and vomiting. She developed blurred vision and left side weakness three days after the onset of headache.

Except for episodes of migraine headache, which were manageable with first-line analgesics, her medical or family history was unremarkable. Her family history did not indicate any potential prothrombotic conditions.

On arrival at the hospital, she was awake and oriented with a Glasgow Coma Scale (GCS) of 15 out of 15. Her vital signs and oxygen saturation were within normal limits. A neurological examination demonstrated bilateral papilledema, left-sided hemianopia, and left-sided hemiparesis. The plantar reflex was extensor on the left side. 
Initial blood studies showed the following: white cell $\left(8 \times 10^{3} / \mu 1\right.$, with lymphocyte count $7.7 \%$,

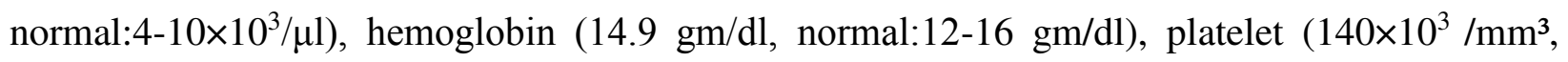
normal:150-450 $\left.\times 10^{3} . / \mathrm{mm}^{3}\right)$, C-reactive protein (CRP) (40mg/l, normal: 0-6 mg/l), erythrocyte sedimentation rate (ESR) $(12 \mathrm{~mm} / \mathrm{hr}$, normal:0-30 mm/hr), lactate dehydrogenase (LDH) (751 u/l, normal: <480 u/l ), ferritin level (144 ng/ml, normal: 4-204 ng/ml), D-dimer (>10000 ng/ml FEU, normal: up to $500 \mathrm{ng} / \mathrm{ml} \mathrm{FEU),} \mathrm{and} \mathrm{normal} \mathrm{liver} \mathrm{function} \mathrm{test} \mathrm{(LFT).} \mathrm{Viral} \mathrm{markers} \mathrm{including}$ human immunodeficiency virus (HIV), the hepatitis C virus (HCV) antibody and the hepatitis B surface antigen ( $\mathrm{HBs} \mathrm{Ag}$ ) were negative.

Due to the COVID-19 pandemic and elevated CRP, real-time reverse-transcriptase polymerase chain reaction (RT-PCR) for SARS-CoV-2 using oropharyngeal/nasopharyngeal swabs and lung high-resolution computed tomography (HRCT) was performed. RT-PCR was positive and lung HRCT revealed bilateral lower lobes subpleural bands.

Spiral brain CT demonstrated a hemorrhagic venous infarct in the right parieto-occipital area associated with dense clot sign in the posterior part of the superior sagittal sinus (Fig.1A). Brain MRI and MRV confirmed the diagnosis of CVST with displaying widespread thromboses in cortical veins, superior sagittal, transverse, and sigmoid sinuses (Fig.1B-D). Consequently, the patient was promptly treated with unfractionated heparin 1000 units/hr as a continuous IV infusion, acetazolamide $250 \mathrm{mg}$ P.O. twice daily, furosemide $20 \mathrm{mg}$ P.O. twice daily, hypertonic saline ( $\mathrm{NaCl} 5 \%$ ) 1 vial IV q8h,-lopinavir/ritonavir 400/100 mg P.O. twice daily, and dexamethasone 8 mg IV once daily.

On the $4^{\text {th }}$ day of admission, despite receiving a therapeutic dose of anticoagulant, she was still suffering from a severe headache and developed an altered mental status. The subsequent brain CT showed slight hematoma expansion and aggravation of the brain edema. Intrasinus thrombolysis and mechanical thrombectomy were considered for her. Recombinant tissue plasminogen activator (rTPA) $20 \mathrm{mg}$ was infused slowly into the superior sagittal sinus followed by manual thrombosuction with a satisfactory result. However, despite the initial recanalization, her clinical condition deteriorated within the next two days. She developed a decreased level of consciousness (GCS 8 out of 15) and gasping respiration. Afterward, she developed sustained metabolic acidosis. Mesenteric artery thrombosis could be one of the differential diagnoses; however, mesenteric artery CT angiography could not be performed owing to her poor condition. 
On the $7^{\text {th }}$ day of admission, she became febrile $\left(\mathrm{T}: 38.5^{\circ} \mathrm{c}\right)$ and blood culture was positive for enterobacter species. Ampisulbactam 3 gr IV q8h and vancomycin 1 gr IV q12h were administered. Due to the right-sided leg swelling and significant size difference between right and left calves' circumference, a color doppler ultrasound of both lower extremities was performed which revealed partial thrombosis of the right saphenofemoral vein.

Hypercoagulable workup was substantial for elevated lupus anticoagulant (>120 seconds, normal: 28-45 seconds), positive anti-phospholipid IgM antibody (1.3, normal: $<1.2)$, antinuclear antibody $(1.5$, normal:<1.2), and anti-double-stranded DNA (27 IU/ml, normal:<18 IU/ml). The other vasculitis and hypercoagulable markers were normal.

The brain CT on the $8^{\text {th }}$ day demonstrated evidence of a midline shift to the left side about $10 \mathrm{~mm}$. She underwent a decompressive craniectomy. On the ninth day of admission, she became hypotensive and had coffee-ground nasogastric tube drainage. Blood investigation showed leukocytosis $\left(13.8 \times 10^{3} / \mathrm{ul}\right)$, significant hemoglobin drop (from 14.9 to $10.3 \mathrm{gm} / \mathrm{dl}$ ), thrombocytopenia $\left(65 \times 10^{3} / \mathrm{mm}^{3}\right)$, elevated partial thromboplastin time (159 seconds, normal:2536 seconds), and prothrombin time (22.8, normal:10-13 seconds) as well as low fibrinogen level (102 mg/dl, normal: 200-400mg/dl). The putative differential diagnosis was septic shock and disseminated intravascular coagulation (DIC). She died on the $10^{\text {th }}$ day of admission.

\section{Discussion}

This study represents a case series of CVST associated with SARS-CoV-2 infection, diagnosed and treated at multiple institutions throughout Iran. We provided a brief overview of all previously reported cases in Table 2 as well [6-18].

Several months after the spread of SARS-CoV-2, the association of the infection with thromboembolic events such as cerebrovascular disease seems to be stronger than considered incidental. Whether SARS-CoV-2 infection "triggers" the coagulation cascade in patients with other underlying diseases or directly "causes" CVST remains to be elucidated [23]. Since $48 \%$ of the cumulative data group had other predisposing factors for CVST, the role of SARS-CoV-2 as a principal contributor versus an additive factor is still unclear. 
The mean age of the patients in the cumulative data group is greater than the former reports of CVST without SARS-CoV-2 infection. Since older patients seem to be more susceptible to SARSCoV-2 infection [24], the mean age of CVST patients is expected to be higher in the setting of SARS-CoV-2 infection. Meanwhile, the proportion of women was much higher in the former reports of CVST without COVID-19. Given that men are more vulnerable to the severe consequences of COVID-19 [25] and as mentioned before, the incidence of thrombotic events is greater in seriously affected patients with COVID-19 [26], a reduction in the dominance of women over men in the context of SARS-CoV-2 infection might be reasonable.

Besides, three patients of the current study and three of the previously reported cases developed other thrombotic events such as deep vein thrombosis, pulmonary thromboembolism, or arterial thrombosis, simultaneously with CVST or during hospitalization. It might be due to a higher load of thrombophilia caused by SARS-CoV-2 [23].

The mortality rate in CVST associated with SARS-CoV-2 infection is higher (35.29\%) than the former reports of CVST (shown in Table 3). A higher burden of thrombosis, the involvement of several dural sinuses, and the wide range of COVID-19 complications [27] may contribute to the worse outcomes in these patients. These complications demand a high level of vigilance from physicians about CVST in COVID-19 patients.

The lack of respiratory symptoms of COVID-19 has been observed in four of our patients and four of the previously reported cases, indicating the absence of correlation between the presence of the respiratory symptoms and the risk of developing CVST. Besides, in five cases of the current series and three of the previously reported cases, there was no gap between the diagnosis of COVID-19 and CVST, suggesting that CVST might be the primary manifestation of COVID-19. Therefore, a high level of suspicion of SARS-CoV-2 infection is essential in all patients with CVST within the pandemic.

Endovascular intervention was performed for one of our patients (case no.1 in Table 1) and one of the patients reported by Cavalcanti et al. [10] with relative success and recanalization of the thrombosed sinuses; however, the patients' outcomes were not changed and both patients died.

As limitations of the current study, some inflammatory or coagulation biomarkers of the patients were missing. This can be justified because of the dismal clinical condition of some of our patients. 
Meanwhile, we did not have more accurate diagnostic modalities than brain CT scan to diagnose CVST due to unanticipated death in two patients. As a major drawback, this is a descriptive noncase-controlled study and these results should be confirmed by case-control studies. It also should be kept in mind that the association could have been a coincidence instead of a risk factor in some patients.

In conclusion, SARS-CoV-2 can induce thrombosis in patients with or without conventional risk factors for CVST. In the COVID-19 pandemic era, a high index of suspicion of CVST should be considered [28], particularly in patients with headache, focal neurological deficit, and encephalopathy; whether they present respiratory symptoms of SARS-CoV-2 infection or not.

\section{Funding}

None.

\section{Compliance with ethical standards}

Conflicts of interest The authors declare that they have no conflict of interest.

\section{Supplemental Materials}

Clinical vignettes of the case no.2-6.

\section{Author Contributions}

All authors contributed to the study conception and design. Material preparation, data collection, and analysis were performed by MAD, ME, AAB, ZB, FF, HM, ARJ, MP, NF, MB, MO, MSS, SSZ, FM, AS, SS, AM, MRA, and RZ. The first draft of the manuscript was written by ABH, EH, VRO, RF, MR, and all authors commented on previous versions of the manuscript. All authors read and approved the final manuscript. 


\section{References}

1. Wasay M, Kaul S, Menon B, Dai AI, Saadatnia M, Malik A, et al. Asian Study of Cerebral Venous Thrombosis. J Stroke Cerebrovasc Dis.: the official journal of National Stroke Association. 2019;28(10):104247.

2. Ashjazadeh N, Borhani Haghighi A, Poursadeghfard M, Azin H. Cerebral venous-sinus thrombosis: a case series analysis. Iran J Med Sci. 2011;36(3):178-82.

3. Borhani Haghighi A, Edgell RC, Cruz-Flores S, Feen E, Piriyawat P, Vora N, et al. Mortality of cerebral venous-sinus thrombosis in a large national sample. Stroke. 2012;43(1):2624.

4. Borhani Haghighi A, Ashjazadeh N, Safari A, Cruz-Flores S. Cerebral venous sinus thrombosis in iran: cumulative data, shortcomings and future directions. Iran. Red Crescent Med. J. 2012;14(12):805-10.

5. Gklinos P. Neurological manifestations of COVID-19: a review of what we know so far. J Neurol. 2020;26:1-5.

6. Baudar C, Duprez T, Kassab A, Miller N, Rutgers MP. COVID-19 as triggering co-factor for cortical cerebral venous thrombosis? J Neuroradiol. 2020;S0150-9861(20)30205-4.

7. Klein DE, Libman R, Kirsch C, Arora R. Cerebral venous thrombosis: A typical presentation of COVID-19 in the young. J Stroke Cerebrovasc Dis.: the official journal of National Stroke Association. 2020;29(8):104989.

8. Garaci F, Di Giuliano F, Picchi E, Da Ros V, Floris R. Venous cerebral thrombosis in COVID-19 patient. J Neurol Sci. 2020;414:116871.

9. Hughes C, Nichols T, Pike M, Subbe C, Elghenzai S. Cerebral Venous Sinus Thrombosis as a Presentation of COVID-19. Eur J Case Rep Intern Med. 2020;7(5):001691.

10. Cavalcanti DD, Raz E, Shapiro M, Dehkharghani S, Yaghi S, Lillemoe K, et al. Cerebral Venous Thrombosis Associated with COVID-19. AJNR Am J Neuroradiol. 2020;41(8):1370-6.

11. Hemasian H, Ansari B. First case of Covid-19 presented with cerebral venous thrombosis: A rare and dreaded case. Rev Neurol (Paris). 2020;176(6):521-3.

12. Poillon G, Obadia M, Perrin M, Savatovsky J, Lecler A. Cerebral venous thrombosis associated with COVID-19 infection: Causality or coincidence? J Neuroradiol. 2020; S01509861(20)30167-X.

13. Malentacchi M, Gned D, Angelino V, Demichelis S, Perboni A, Veltri A, et al. Concomitant brain arterial and venous thrombosis in a COVID-19 patient. Eur J Neurol. 2020; 10.1111/ene.14380.

14. Bolaji P, Kukoyi B, Ahmad N, Wharton C. Extensive cerebral venous sinus thrombosis: a potential complication in a patient with COVID-19 disease. BMJ Case Rep. 2020;13(8):e236820.

15. Roy-Gash F, De Mesmay M, Devys JM, Vespignani H, Blanc R, Engrand N. COVID-19associated acute cerebral venous thrombosis: clinical, CT, MRI and EEG features. Crit Care. 2020;24(1):419. 
16. Aghayari Sheikh Neshin S, Basirjafari S, Saberi A, Shahhosseini B, Zarei M. Liver abnormality may develop cerebral vein thrombosis in COVID-19. J Neurol Sci. 2020;417:117076. 17. Sugiyama Y, Tsuchiya T, Tanaka R, Ouchi A, Motoyama A, Takamoto T, et al. Cerebral venous thrombosis in COVID-19-associated coagulopathy: A case report. J Clin Neurosci. 2020;79:30-2.

18. Mowla A, Shakibajahromi B, Shahjouei S, Borhani-Haghighi A, Rahimian N, Baharvahdat $\mathrm{H}$, et al. Cerebral venous sinus thrombosis associated with SARS-CoV-2; a multinational case series. J Neurol Sci. 2020:117183.

19. Ellul MA, Benjamin L, Singh B, Lant S, Michael BD, Easton A, et al. Neurological associations of COVID-19. Lancet Neurol. 2020;19(9):767-83.

20. Saposnik G, Barinagarrementeria F, Brown Jr RD, Bushnell CD, Cucchiara B, Cushman $\mathrm{M}$, et al. Diagnosis and management of cerebral venous thrombosis: a statement for healthcare professionals from the American Heart Association/American Stroke Association. Stroke. 2011;42(4):1158-92.

21. Ferro JM, Canhão P, Stam J, Bousser MG, Barinagarrementeria F. Prognosis of cerebral vein and dural sinus thrombosis: results of the International Study on Cerebral Vein and Dural Sinus Thrombosis (ISCVT). Stroke. 2004;35(3):664-70.

22. Shakibajahromi B, Haghighi AB, Salehi A, Vardanjani HM, Ghaedian M, Safari A, et al. Clinical and radiological characteristics and predictors of outcome of cerebral venous sinus thrombosis, a hospital-based study. Acta Neurol Belg. 2020;120(4):845-52.

23. Bhatia R, Srivastava MVP. COVID-19 and Stroke: Incidental, Triggered or Causative. Ann Indian Acad Neurol. 2020;23(3):318-24.

24. Zhang J, Wang X, Jia X, Li J, Hu K, Chen G, et al. Risk factors for disease severity, unimprovement, and mortality in COVID-19 patients in Wuhan, China. Clin Microbiol Infect. 2020;26(6):767-72.

25. Goyal P, Choi JJ, Pinheiro LC, Schenck EJ, Chen R, Jabri A, et al. Clinical Characteristics of Covid-19 in New York City. N Engl J Med. 2020;382(24):2372-4.

26. Klok FA, Kruip M, van der Meer NJM, Arbous MS, Gommers D, Kant KM, et al. Confirmation of the high cumulative incidence of thrombotic complications in critically ill ICU patients with COVID-19: An updated analysis. Thromb Res. 2020;191:148-50.

27. McIntosh K. Coronavirus disease 2019 (COVID-19): Epidemiology, virology, clinical features, diagnosis, and prevention. Uptodate 2020.

28. Shakibajahromi B, Borhani-Haghighi A, Haseli S, Mowla A. Cerebral venous sinus thrombosis might be under-diagnosed in the COVID-19 era. eNeurologicalSci. 2020;20:100256. 


\section{Figure Legend}

Fig. 1 (A) Axial non-enhanced spiral brain computed tomography (CT) scan shows a large hematoma with surrounding edema in the right parieto-occipital lobe (black thin arrows); Note the dense clot sign in the posterior part of the superior sagittal sinus, characteristic of thrombosis (white thick arrow). (B) Axial T2-weighted magnetic resonance (MR) image shows a large heterogeneous lesion with surrounding edema in the right parieto-occipital lobe (white thin arrows) associated with loss of signal void in the posterior region of the superior sagittal sinus (white thick arrow) suggestive of the venous sinus thrombosis with hemorrhagic venous infarction. (C) Axial contrast-enhanced T1-weighted MR image shows a filling defect in the posterior part of the superior sagittal sinus suggestive of thrombosis (white thick arrow). (D) Brain MR venography shows extensive thrombosis in the superior sagittal, right transverse, and sigmoid sinuses as well as the right internal jugular vein (white thin arrows). 


\section{Figures}

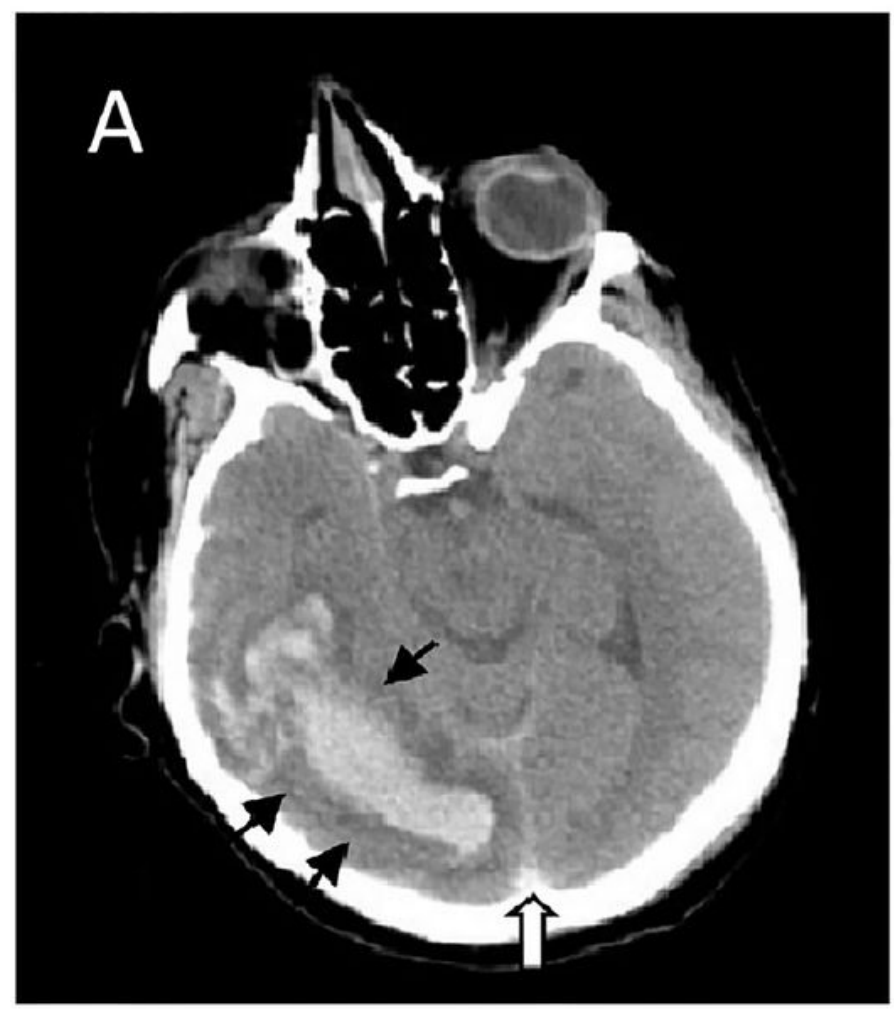

\section{B}

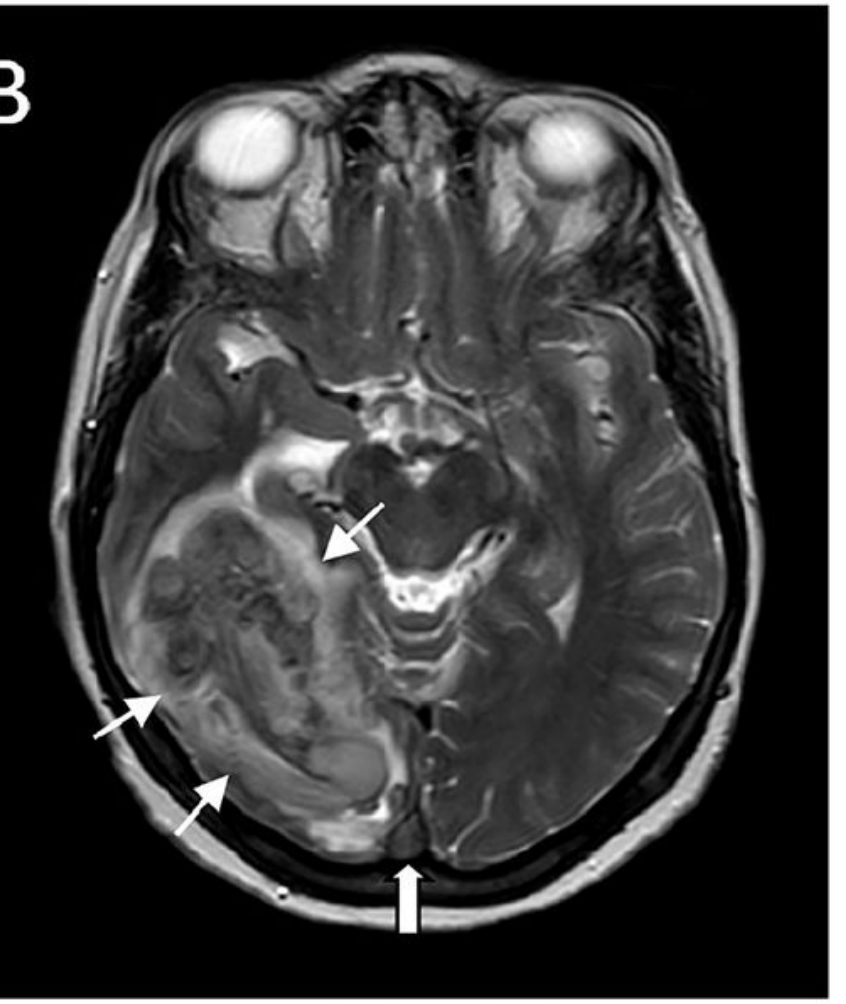

C
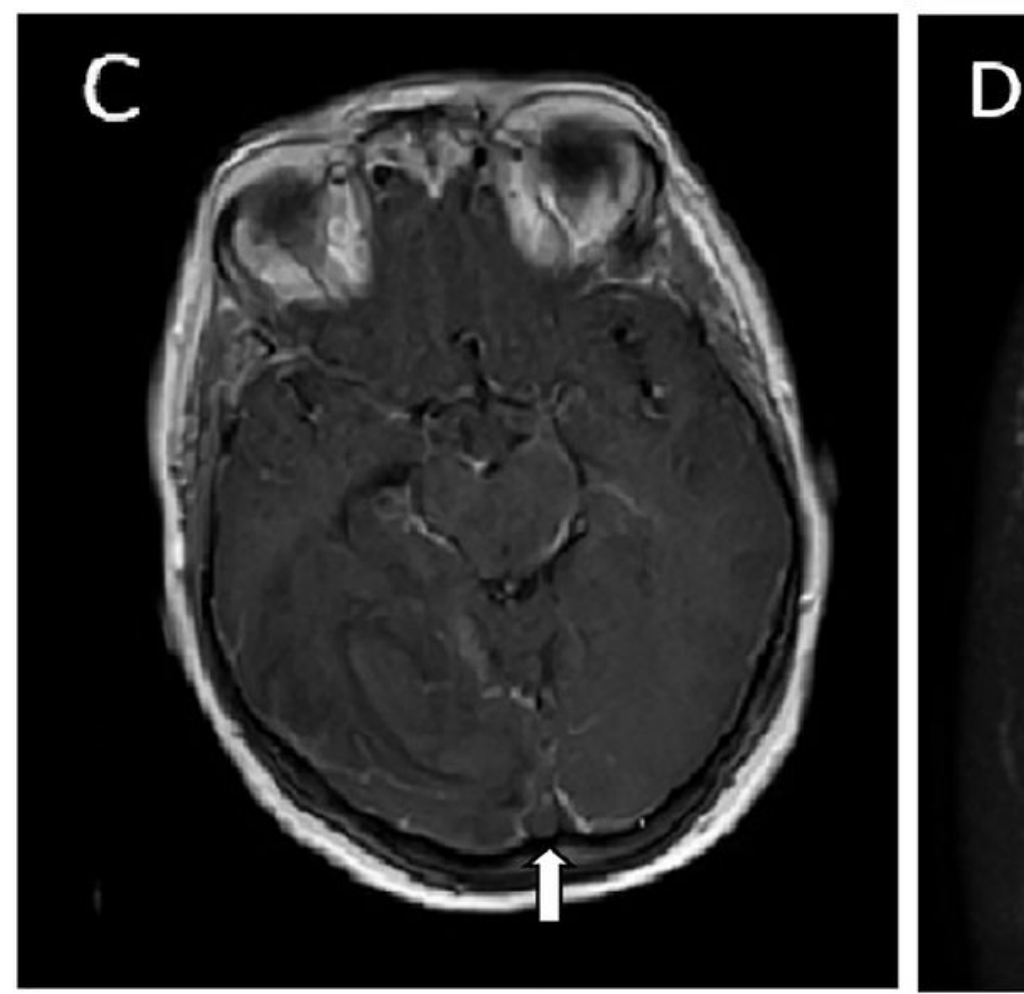

D

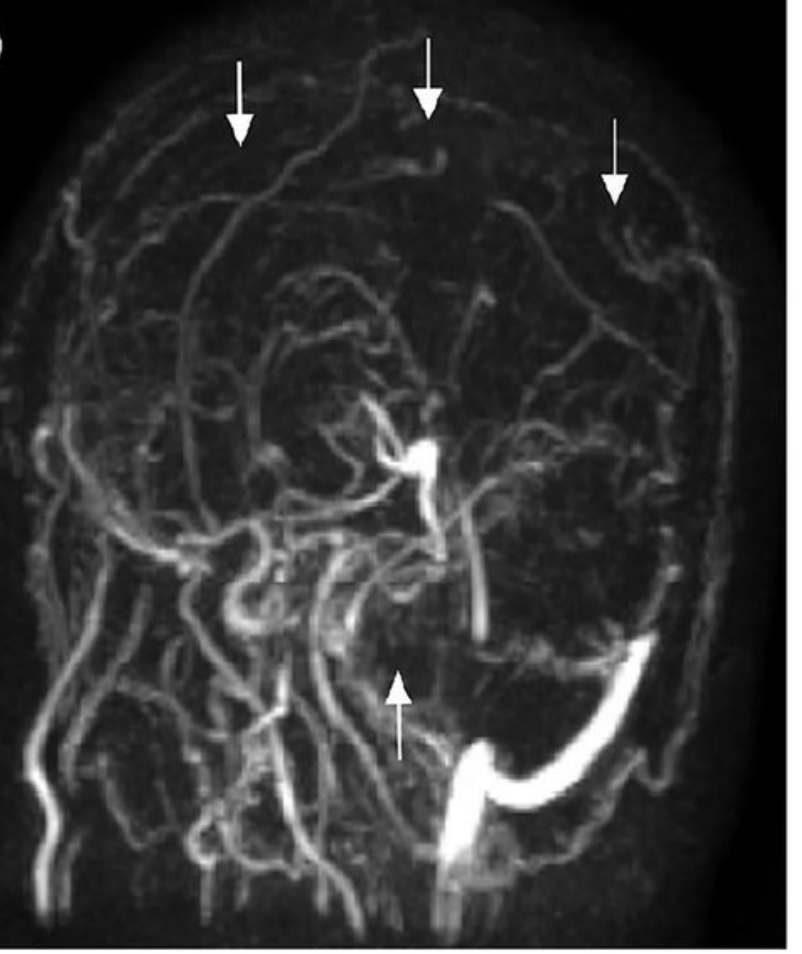

Figure 1

(A) Axial non-enhanced spiral brain computed tomography (CT) scan shows a large hematoma with surrounding edema in the right parieto-occipital lobe (black thin arrows); Note the dense clot sign in the posterior part of the superior sagittal sinus, characteristic of thrombosis (white thick arrow). (B) Axial T2- 
weighted magnetic resonance (MR) image shows a large heterogeneous lesion with surrounding edema in the right parieto-occipital lobe (white thin arrows) associated with loss of signal void in the posterior region of the superior sagittal sinus (white thick arrow) suggestive of the venous sinus thrombosis with hemorrhagic venous infarction. (C) Axial contrast-enhanced T1-weighted MR image shows a filling defect in the posterior part of the superior sagittal sinus suggestive of thrombosis (white thick arrow). (D) Brain MR venography shows extensive thrombosis in the superior sagittal, right transverse, and sigmoid sinuses as well as the right internal jugular vein (white thin arrows).

\section{Supplementary Files}

This is a list of supplementary files associated with this preprint. Click to download.

- SupplementaryCaseNo.26.pdf

- Table1.pdf

- Table2.pdf

- Table3.pdf 